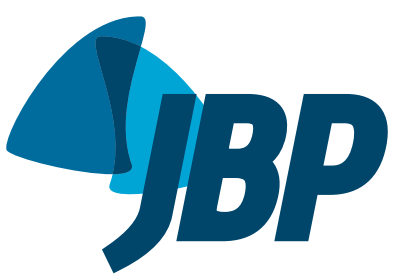

\section{Serum pyrazinamide concentrations in patients with pulmonary tuberculosis}

\author{
Stefania de Medeiros Araujo Lucena ${ }^{1, a}$, Carlos Augusto Abreu Alberio ${ }^{2, b}$, \\ Ana Carla Godinho Pinto ${ }^{3, c}$, José Luiz Fernandes Vieira ${ }^{2,4, d}$
}

\section{TO THE EDITOR,}

Tuberculosis is an important public health issue in Brazil. Although incidence rates have decreased and cure rates have increased in recent years in the country, there were 69,569 reported new cases of tuberculosis in 2017..$^{(1,2)}$ Exposing Mycobacterium tuberculosis to effective serum concentrations of chemotherapeutic agents is a requirement for maintaining and increasing the cure rates, as well as for reducing the risk of the emergence of drug-resistant bacilli. ${ }^{(3)}$ The measurement of serum concentrations of chemotherapeutic agents is considered the gold standard for assessing M. tuberculosis exposure to such drugs. However, it has not been widely used in patients with tuberculosis, most studies having focused on measuring concentrations of rifampin and isoniazid. ${ }^{(4)}$ There have been few studies investigating the serum concentrations of pyrazinamide achieved after the use of therapeutic doses of the drug, which might be due to the fact that pyrazinamide is used only in the intensive phase of tuberculosis treatment.

Pyrazinamide is a prodrug that requires conversion to its active metabolite, pyrazinoic acid, by pyrazinamidase, an M. tuberculosis enzyme. Its therapeutic efficacy depends on the serum concentrations achieved after administration of conventional doses; for tuberculosis treatment, doses should range from 20 to $50 \mu \mathrm{g} / \mathrm{mL} .^{(3,4)}$ However, there is wide interindividual variation in serum pyrazinamide concentrations during the course of tuberculosis treatment, which requires that these concentrations be periodically monitored in studies of therapeutic efficacy. ${ }^{(4,5)}$ In addition, there have been few studies investigating the influence of gender and treatment duration on serum pyrazinamide concentrations. Finally, there is lack of studies investigating M. tuberculosis exposure to pyrazinamide in Brazil. To clarify these issues, we assessed serum pyrazinamide concentrations in 46 patients over 18 years of age with a clinical and laboratory diagnosis (positive sputum smear and culture results) of tuberculosis caused by M. tuberculosis. Patients were selected from two health care clinics in the city of Belém, Brazil, where their antituberculosis treatment was initiated. We excluded cases of retreatment, treatment dropout, multidrug-resistant tuberculosis, and extrapulmonary tuberculosis, as well as pregnant women, breastfeeding women, individuals with comorbidities, and licit or illicit drug users.

Patients were treated with the standard regimen recommended by the Brazilian National Ministry of Health, which consists of fixed-dose combination tablets containing rifampin (150 mg), isoniazid (75 mg), pyrazinamide (400 $\mathrm{mg})$, and ethambutol (275 $\mathrm{mg})$; treatment duration and dose adjustment for patient weight were as per the guidelines. ${ }^{(2)}$ Administration was once daily, preferably on an empty stomach, and in the presence of a family member. Clinical and laboratory follow-up lasted six months. Blood samples were collected before the initiation of therapy, as well as before and after drug administration (pre-dose and post-dose samples, respectively) on treatment days 30 and 60 , which corresponded to the days when patients returned to the clinic to get their free monthly medication. On those days, patients reported to the clinic with an empty stomach for supervised administration of the dose by the project team. Serum pyrazinamide concentrations were measured by high-performance liquid chromatography. ${ }^{(6)}$

Serum pyrazinamide concentrations were expressed as median (interquartile range) and were compared between sample collection days (day 30 vs. day 60), as well as between genders, with the Mann-Whitney $U$ test. The level of significance required in order to reject the null hypothesis was set at $5 \%$. The study was approved by the Human Research Ethics Committee of the Federal University of Pará Center for Tropical Medicine (Protocol no. 1.591.019).

All 46 patients completed the clinical and laboratory follow-up and had a negative sputum smear at the end of the second month of treatment. None of the samples collected at patient inclusion $(n=46)$ showed measurable concentrations of pyrazinamide, which indicates that the drug had not been used recently. The time between drug administration and the collection of pre-dose samples on days 30 and 60 ( $n=46$ for both time points) ranged from 22 to $24 \mathrm{~h}$; and the time between drug administration on days 30 and 60 and the collection of post-dose samples ( $n=25$ for both time points) ranged from 40 to $70 \mathrm{~min}$. In the pre-dose samples, the median plasma concentrations of pyrazinamide were $3.75 \mu \mathrm{g} /$ $\mathrm{mL}(0.3-10.9 \mu \mathrm{g} / \mathrm{mL})$ and $2.7 \mu \mathrm{g} / \mathrm{mL}(0.4-10.7 \mu \mathrm{g} / \mathrm{mL})$ on days 30 and 60 , respectively. Serum pyrazinamide concentrations were similar at the two study time points $(U=890 ; p=0.191)$.

Given the pharmacokinetic properties of pyrazinamide, such as its biological half-life of 8 to $11 \mathrm{~h}$, the pyrazinamide concentrations present in pre-dose samples are of limited value in assessing exposure to the drug, because

\footnotetext{
1. Programa de Pós-Graduação em Ciências Farmacêuticas, Universidade Federal do Pará, Belém (PA) Brasil.

2. Programa de Pós-Graduação em Doenças Tropicais, Universidade Federal do Pará, Belém (PA) Brasil.

3. Instituto de Ciências da Saúde, Universidade Federal do Pará, Belém (PA) Brasil.

4. Núcleo de Doenças Tropicais, Universidade Federal do Pará, Belém (PA) Brasil.

a. (iD) http://orcid.org/0000-0003-1865-3264; b. (iD) http://orcid.org/0000-0002-9364-3990; c. (iD) http://orcid.org/0000-0001-7498-8865;

d. (iD) http://orcid.org/0000-0003-4842-8762
} 
(A)

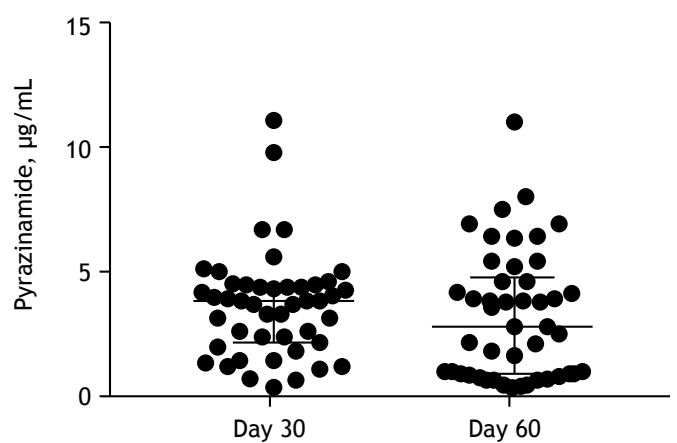

(B)

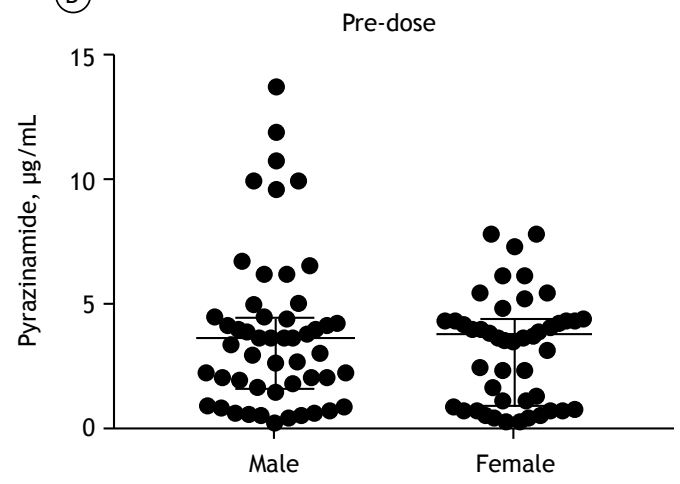

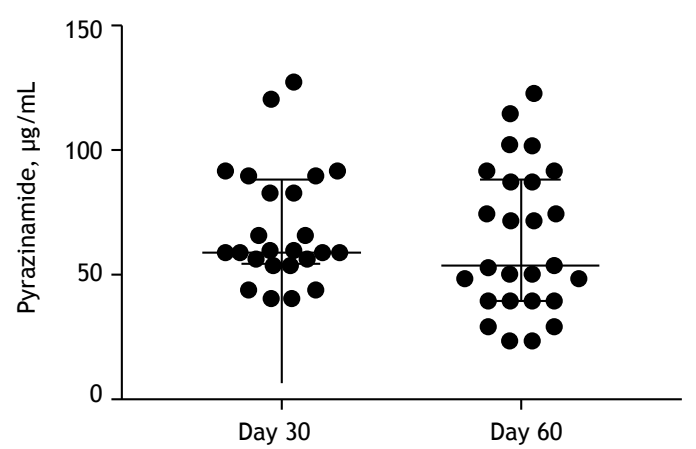

Post-dose

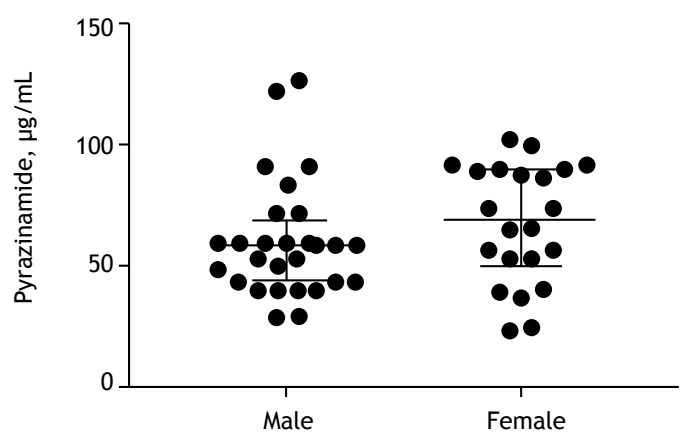

Figure 1. Serum pyrazinamide concentrations measured in blood samples collected before and after supervised drug administration (pre-dose and post-dose samples, respectively). Comparison between sample collection days (day 30 vs. day 60; in A) and between genders (in B). The longest horizontal line represents the median of the results, and the other horizontal lines represent the interquartile ranges.

they correspond to residual values. ${ }^{(5)}$ In addition, pyrazinamide does not accumulate significantly in organic compartments, which can be verified by the low pyrazinamide concentrations present in pre-dose samples, as well as by the absence of significant differences in the serum pyrazinamide concentrations of samples collected on days 30 and 60 .

Post-dose samples more reliably indicate bacillus exposure to drugs, especially drugs with a short biological half-life. ${ }^{(5)}$ In addition, assessment of exposure to this type of drug can be performed on any treatment day. ${ }^{(5)}$ In the present study, the median plasma concentrations of pyrazinamide were $59.5 \mu \mathrm{g} / \mathrm{mL}$ $(40.3-127.0 \mu \mathrm{g} / \mathrm{mL})$ and $53.6 \mu \mathrm{g} / \mathrm{mL}(23.4-122.7 \mu \mathrm{g} /$ $\mathrm{mL}$ ) on days 30 and 60 , respectively (Figure $1 \mathrm{~A}$ ). Serum pyrazinamide concentrations were similar at the two study time points $(U=253 ; p=0.254)$. These results are within the range of effective serum concentrations for pyrazinamide-susceptible $M$. tuberculosis strains $(20.0-50.0 \mu \mathrm{g} / \mathrm{mL}) .^{(3-5)}$ In fact, serum concentrations below $20 \mu \mathrm{g} / \mathrm{mL}$ are considered low and suggest a change in the oral bioavailability of the drug, as well as being associated with increased treatment failure..$^{(3-5)}$ Therefore, the serum pyrazinamide concentrations found in the patients included in the present study indicate that the treatment regimen used in Brazil provides adequate bacillus exposure to the drug. This finding was corroborated by the fact that all patients were smear negative at the end of the intensive phase of treatment. There was a wide range of pyrazinamide concentrations in the pre-dose and post-dose samples. That finding has been reported in studies of pyrazinamide pharmacokinetics and has been related to variations in absorption and clearance. In addition, it is likely that the time between drug administration and the collection of blood samples contributed to the variation in the drug concentrations. ${ }^{(4,5)}$ In fact, it has been reported that serum pyrazinamide concentrations measured in blood samples collected $2 \mathrm{~h}$ after its administration more reliably reflect the end of the absorption phase and show less interindividual variation. ${ }^{(3,5)}$ However, it is likely that the time to sample collection in the present study did not significantly influence the assessment of M. tuberculosis exposure to the drug, given that the values found were similar to those reported in studies of other population groups. ${ }^{(3-5)}$

Gender influences serum concentrations of several chemotherapeutic agents because of differences in hormone concentrations and in body fat distribution. (5) The medians (interquartile ranges) of the serum pyrazinamide concentrations in the pre-dose samples from men $(n=25)$ and women $(n=21)$ were $3.7 \mu \mathrm{g} /$ $\mathrm{mL}(0.3-13.9 \mu \mathrm{g} / \mathrm{mL})$ and $3.8 \mu \mathrm{g} / \mathrm{mL}(0.3-7.9 \mu \mathrm{g} / \mathrm{mL})$, respectively (Figure $1 \mathrm{~B})$. In the post-dose samples from men $(n=14)$ and women $(n=11)$, the medians (interquartile ranges) of the serum pyrazinamide 
concentrations were $59 \mu \mathrm{g} / \mathrm{L}(29.5-127.0 \mu \mathrm{g} / \mathrm{mL})$ and $70.1 \mu \mathrm{g} / \mathrm{mL}(23.4-102.5 \mu \mathrm{g} / \mathrm{mL})$, respectively (Figure $1 B)$. In the present study, gender did not significantly influence the pre-dose pyrazinamide concentrations $(U=112 ; p=0.659)$ or post-dose pyrazinamide concentrations $(U=250 ; p=0.261)$.

The main limitations of this study were the small sample size and the time of collection of the post-dose samples, which might have contributed to the variation in serum pyrazinamide concentrations among the patients. Another limitation is that the chemotherapeutic agents were administered under the supervision of the health care team only on the sample collection days; on the remaining days, they were administered under the supervision of a family member, which does not ensure strict adherence to the treatment regimen, although all of the patients had a negative sputum smear at the end of the second month of treatment.

In conclusion, the results of the present study confirm that the tuberculosis treatment regimen adopted by the Brazilian National Ministry of Health provides adequate $M$. tuberculosis exposure to pyrazinamide. In addition, they show that gender and the timing of blood sample collection did not influence serum pyrazinamide concentrations.

\section{REFERENCES}

1. Brasil. Ministério da Saúde. Secretaria de Vigilância em Saúde Boletim Epidemiológico 11. Implantação do Plano Nacional pelo Fim da Tuberculose como Problema de Saúde Pública no Brasil: primeiros passos rumo ao alcance das metas. Brasília: Ministério da Saúde; 2018.

2. Brasil. Ministério da Saúde. Secretaria de Vigilância em Saúde. Departamento de Vigilância Epidemiológica. Manual de Recomendações para o Controle da Tuberculose no Brasil. Série A. Normas e Manuais Técnicos. Brasília: Ministério da Saúde; 2011.

3. Park JS, Lee JY, Lee YJ, Kim SJ, Cho YJ, Yoon HI. Serum Levels of Antituberculosis Drugs and Their Effect on Tuberculosis Treatment Outcome. Antimicrob Agents Chemother. 2015;60(1):92-8. https:// doi.org/10.1128/AAC.00693-15
4. Alsultan A, Peloquin CA. Therapeutic drug monitoring in the treatment of tuberculosis: an update. Drugs. 2014;74(8):839-54. https://doi.org/10.1007/s40265-014-0222-8

5. Chirehwa MT, Mcllleron H, Rustomjee R, Mthiyane T, Onyebujoh P. Smith P. Pharmacokinetics of Pyrazinamide and Optimal Dosing Regimens for Drug-Sensitive and -Resistant Tuberculosis. Antimicrob Agents Chemother. 2017;61(8). pii: e00490-17. https:// doi.org/10.1128/AAC.00490-17

6. Prasanthi B, Ratna JV, Phani RSC. Development and validation of RP-HPLC method for simultaneous estimation of rifampicin, isoniazid and pyrazinamide in human plasma. J Anal Chem. 2015;70(8):10151022. https://doi.org/10.1134/S1061934815080146 